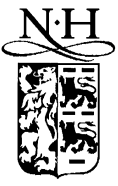

ELSEVIER

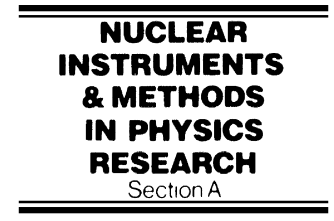

www.elsevier.nl/locate/nima

\title{
The intermediate silicon layers detector at CDFII: Design and progress
}

A. Affolder ${ }^{\mathrm{a}}$, P. Azzi-Bacchetta ${ }^{\mathrm{b}}$, N. Bacchetta ${ }^{\mathrm{b}}$, A. Barbaro-Galtieri ${ }^{\mathrm{a}}$, A. Basti ${ }^{\mathrm{c}}$, F. Bedeschic ${ }^{\mathrm{c}}$ D. Bisello ${ }^{\mathrm{b}}$, S. Blusk ${ }^{\mathrm{d}}$, M. Chertok ${ }^{\mathrm{a}}$, G. Chiarellic ${ }^{\mathrm{c}}$ A. Connolly ${ }^{\mathrm{a}}$, R. Demina ${ }^{\mathrm{b}}$, S. Donati ${ }^{\mathrm{c}}$, R. Ely ${ }^{\mathrm{a}}$, R. Field ${ }^{\mathrm{g}}$, M. Garcia-Sciveres ${ }^{\mathrm{a}}$, J. Goldstein ${ }^{\mathrm{f}}$, G. Grim ${ }^{\mathrm{h}}$, M. Guerzoni ${ }^{\mathrm{i}}$, C. Haber ${ }^{\mathrm{a}}$, K. Hara ${ }^{\mathrm{j}}$, F. Hartmann ${ }^{\mathrm{k}, *}$, A. Heiss ${ }^{\mathrm{k}}$, C. Hill ${ }^{\mathrm{h}}$, M. Hrycyk ${ }^{\mathrm{f}}$, J. Incandela ${ }^{\mathrm{f}}$, Y. Kato ${ }^{1}$, B.J. Kim ${ }^{\mathrm{c}}$, D. Knoblauch ${ }^{\mathrm{k}}$, M. Kruse ${ }^{\mathrm{d}}$, C.M. Lei ${ }^{\mathrm{f}}$, S. Leone ${ }^{\mathrm{c}}$, G. Martignon ${ }^{\mathrm{b}}$, P. Mcyntire ${ }^{\mathrm{e}}$, Y. Miyazaki $^{1}$, A. Moggi ${ }^{\mathrm{c}}$, T. Muller ${ }^{\mathrm{k}}$, A. Munar-Ara ${ }^{\mathrm{c}}$, T. Okusawa ${ }^{1}$, F. Palmonari ${ }^{\mathrm{m}}$, M. Paulini ${ }^{\mathrm{a}}$, D. Pellett ${ }^{\mathrm{h}}$, G. Piacentino ${ }^{\mathrm{m}}$, F. Raffaellic ${ }^{\mathrm{c}}$ D. Saltzberg ${ }^{\mathrm{n}}$, E. Sanders ${ }^{\mathrm{n}}$, M. Schilling ${ }^{\mathrm{k}}$,

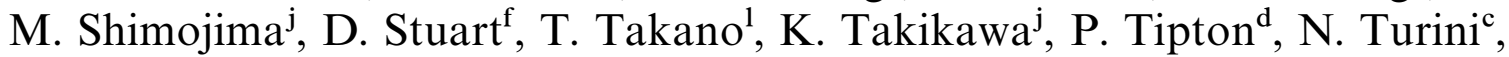
I. Volobouev a , H. Wenzel ${ }^{\mathrm{k}}$, W. Yao ${ }^{\mathrm{a}}$, T. Yoshida ${ }^{1}$, F. Zetti ${ }^{\mathrm{a}}$, S. Zucchelli ${ }^{\mathrm{i}}$

${ }^{a}$ Larwence Berkeley Laboratory, Berkeley, CA, USA

'Universita' and INFN Sez. di Padova, Padova, Italy

'Universita' and INFN Sez. di Pisa, Pisa, Italy

${ }^{\mathrm{d}}$ University of Rochester, Rochester, NY, USA

'Texas A\&M University, USA

${ }^{\mathrm{f}}$ Fermilab, Batavia IL, USA

${ }^{\mathrm{g}}$ University of Florida, USA

${ }^{\mathrm{h}}$ Visiting members to the FNAL group from University of California at Davis, Davis, CA, USA

'Universita' and INFN Sez. di Bologna, Bologna, Italy

${ }^{\mathrm{j}}$ University of Tsukuba, Tsukuba, Japan

${ }^{\mathrm{k}}$ Institut für Experimentelle Kernphysik Universität Karlsruhe, Karlsruhe, Germany

${ }^{1}$ Osaka City University, Osaka, Japan

'Universita' di Cassino, Cassino, Italy

${ }^{\mathrm{n}}$ University of California at Los Angeles, Los Angeles, CA, USA

\section{Abstract}

The Intermediate Silicon Layers Detector is presently being built as part of the CDF upgrades to prepare for the next Tevatron data taking run, scheduled to start in the year 2000. The ISL will be located in the radial region between the Silicon Vertex Detector and the Central Outer Tracker. It will add tracking in the forward region and significantly improve tracking in the central region. Together with the SVX II, the ISL forms a standalone, 3D silicon tracker. In this article we present the design of the ISL and the current status of its construction. (C) 1999 Elsevier Science B.V. All rights reserved.

* Corresponding author.

E-mail address: hartmann@fnal.gov (F. Hartmann) 


\section{Introduction}

The Fermilab Tevatron Collider and its two multipurpose detectors CDF [1] and D0 [2] are preparing for the next data taking run, starting in the year 2000 (RUN II). The goal is the accumulation of $2 \mathrm{fb}^{-1}$ of $\mathrm{p} \overline{\mathrm{p}}$-collisions at $\sqrt{s}=2.0 \mathrm{TeV}$, with instantaneous luminosities of up to $2 \times 10^{32} \mathrm{~cm}^{-2} \mathrm{~s}^{-1}$. The modest increase from 1.8 to $2.0 \mathrm{TeV}$ in the Tevatron center of mass energy leads to a significant increase in cross section of all phenomena, with high mass final states; e.g the $t \overline{\mathrm{t}}$ cross section is increased by $40 \%$. The increase in luminosity, on the other hand, will lead to a 20 -fold increase in event statistics and requires a significant upgrade of the CDF detector.

The CDF II is a general purpose solenoid spectrometer which combines charged particle tracking with projective calorimetry and muon detection. The tracking system is optimized for pattern recognition, momentum resolution, and $b$-tagging up to pseudo rapidity $|\eta| \sim 2$. Combining tracks with information from electromagnetic calorimetry or muon chambers provides efficient electron and muon identification.

\section{Motivation}

The Intermediate Silicon Layers Detector (ISL) (Fig. 1) is an integral part of the CDF II tracking system, which consists of three concentric detector systems. Closest to the beampipe is the Silicon Vertex Detector (SVX II), consisting of five silicon layers at radii between $r=2.45$ and $10.60 \mathrm{~cm}$. The ISL is a silicon detector with a length of almost $2 \mathrm{~m}$, with one central layer $(|\eta|<1)$ at radial position $r=22 \mathrm{~cm}$ and two layers in the forward region $(1<|\eta|<2)$ at radial positions $r=20$ and $28 \mathrm{~cm}$, respectively. The third detector, the Central Outer Tracker (COT), is a large drift chamber with a length of $3 \mathrm{~m}$, covering a radial span beginning at $40 \mathrm{~cm}$ and ending at $137 \mathrm{~cm}$. The ISL provides one or two additional spacepoints in the intermediate region $(r=10.6 \mathrm{~cm}$ up to $40 \mathrm{~cm})$, which improves the linking probability between SVX II tracks and COT tracks, and its fine granularity helps to resolve ambiguities in dense track environments. The complete tracking system, consisting of SVX II, ISL and COT, provides high-momentum resolution for $|\eta|<1\left(\sigma_{P} / P_{\mathrm{T}}^{2}=0.09 \%(\mathrm{GeV} / c)\right.$ at $P_{\mathrm{T}}=10 \mathrm{GeV} / \mathrm{c}$ [1]). In addition, SVX II together with the ISL forms a standalone, 3D silicon tracker, with up to 7 axial and 7 stereo measurements extending the tracking capabilities out to $|\eta| \leq 2$. The $3 \mathrm{D}$ tracking performance and the momentum resolution of the SVX II is largely improved by the ISL and enables good $b$ tagging in the forward region. By increasing the acceptance the ISL has a significant impact on the double $b$-tag efficiency (see Table 1) Fig. 2.

\section{Conceptual design considerations}

The ISL project started in October 1996, the short time available until the insertion scheduled for late 1999 required a quick yet innovative design and aggressive construction schedule. To minimize $R \& D$ and to allow easy integration into CDF, the ISL uses the same readout electronics and data acquisition (DAQ) as the SVX II [1,3]. The design takes advantage of the large radial position; radiation damage occurs more slowly and the hit occupancy is lower, it is therefore possible to use longer strips and a large readout pitch to reduce the number of readout channels and subsequently the cost of frontend electronics and data acquisition. The mechanics of the ISL called for an innovative design. Given the large volume between SVX II and the COT, it was possible to design a light weight yet robust support structure (see Section 4.2). For the ISL we estimate $1.5 \%$ radiation length in the central region and $2 \%$ radiation length in the forward region, including $1 \%$ radiation length due to hybrids, endcaps, cooling.

The result is a detector with 888 large area, double-sided silicon microstrip sensors with a total of 303104 channels. The basic ISL unit is the ladder, composed of three silicon sensors bonded together and their readout electronics located on a hybrid at the end. The support structure is built in carbon fiber. ${ }^{1} 296$ ladders are paired to form 148

\footnotetext{
${ }^{1}$ Testbeam results showed that additional crosstalk with the conductive fiber support and additional capacitance effects can be avoided by isolating the carbon fiber ladder from the sensors and grounding the carbon fiber ladder [4].
} 


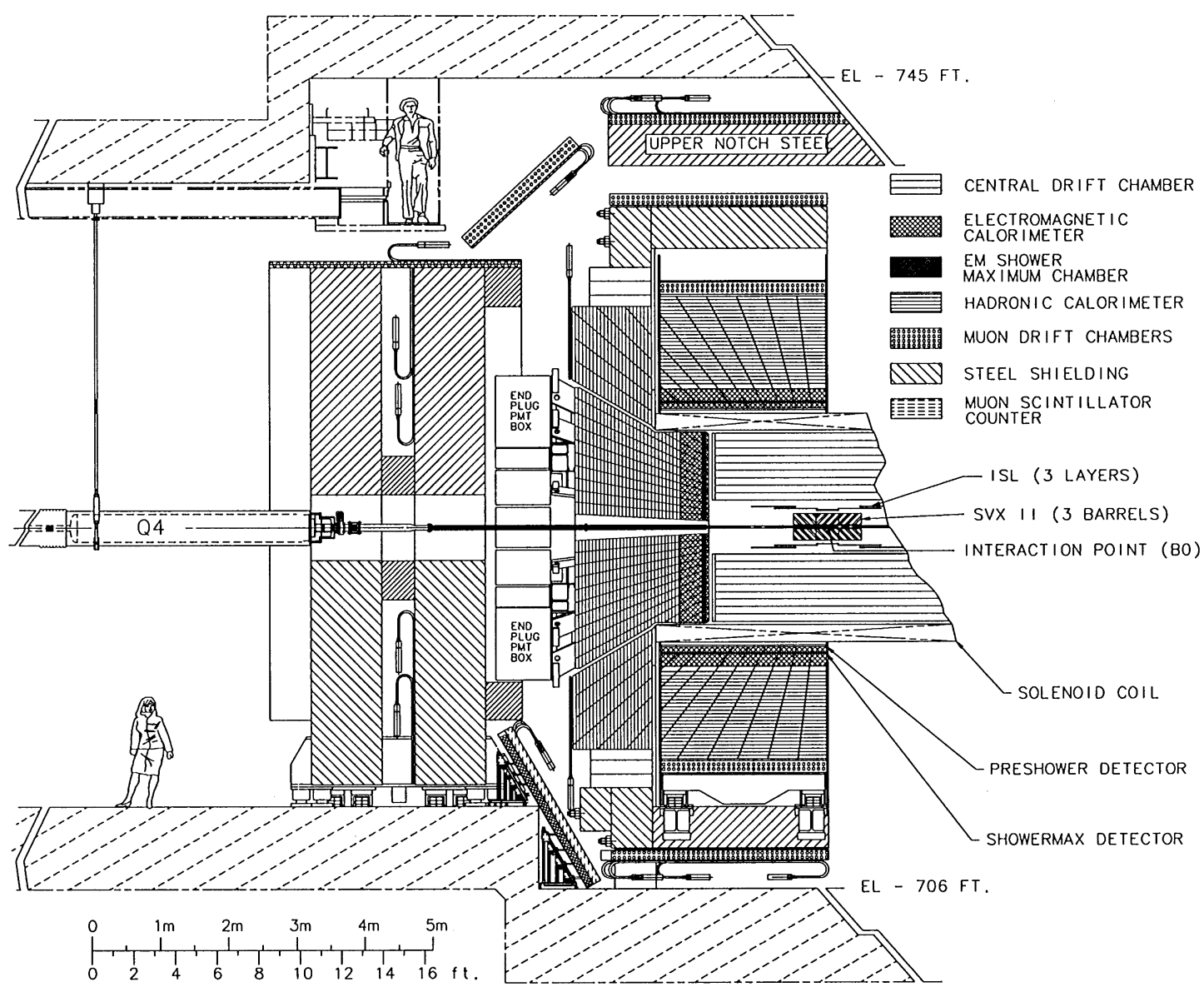

Fig. 1. Elevation view of one-half of the CDF II detector. The tracking system is located inside a superconducting solenoid, which generates a $1.4 \mathrm{~T}$ magnetic field parallel to the beam axis. Calorimetry and muon systems are all outside of the solenoid. The Intermediate Silicon Layers detector (ISL) is located between the inner Silicon Vertex Detector (SVX II) and the Central Outer Tracker (COT).

Table 1

Efficiency for single and double $b$-tagging [1] in ț̄ Monte Carlo events for different detector configurations. The results are from a parametric study, including geometry, acceptance cuts and tracking efficiencies

\begin{tabular}{llr}
\hline $\begin{array}{l}\text { Detector } \\
\text { configuration }\end{array}$ & $\begin{array}{l}\text { Single tag } \\
\text { eff.(\%) }\end{array}$ & \multicolumn{1}{l}{$\begin{array}{l}\text { Double tag } \\
\text { eff.(\%) }\end{array}$} \\
\hline SVX $^{\prime}+$ CTC $^{\text {a }}$ & $37.6 \pm 1.0$ & $6.9 \pm 0.5$ \\
SXV II + COT & $46.7 \pm 1.1$ & $8.7 \pm 0.6$ \\
SVX II + ISL + COT & $60.1 \pm 1.0$ & $15.1 \pm 0.8$ \\
\hline
\end{tabular}

${ }^{\text {a}} \mathrm{SVX}$ ' Silicon Vertex Tracker of CDF for the running period of 1992-1996 (RUN I); CTC Central Tracking Chamber of RUN I. modules of $55 \mathrm{~cm}$ length each. The modules are mounted on carbon fiber rings to form barrels, which are then assembled into a spaceframe (see Section 4.2).

\section{Mechanical components}

\subsection{Ladders and modules}

The most crucial aspect of the construction is the exact alignment of the individual silicon sensors inside the support spaceframe. The internal 




Fig. 2. SVX to SVX II + ISL. SVX was a 4 layer, $51 \mathrm{~cm}$ long detector in RUN I providing $r \phi$ information. It will be replaced by the SVX II-ISL combination; a 5 layer, $87 \mathrm{~cm}$ long detector (SVX II) providing stereo information plus one additional central layer and two additional layers in the forward region (ISL), $195 \mathrm{~cm}$ long. All sensors are double sided with stereo readout, 4 layers with a stereo angle of $1.2^{\circ}$ and 3 with a $90^{\circ}$ stereo angle; the rectangles on the right represent the different layers and their strip layout.

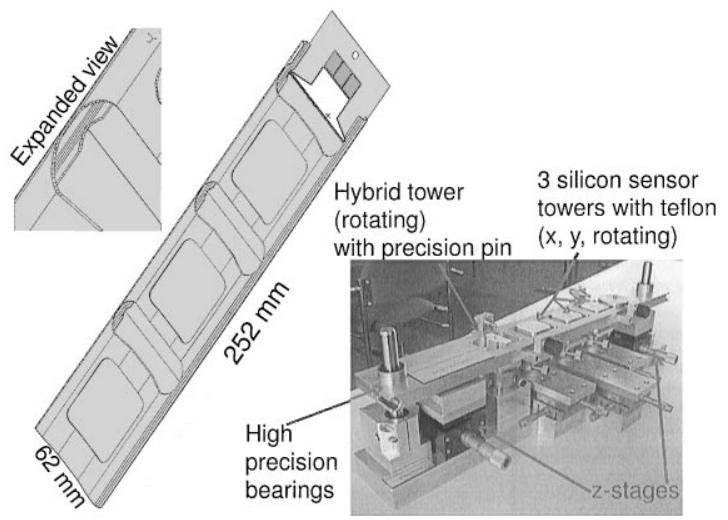

Fig. 3. ISL ladder design and mounting jig. The picture on the left shows the design of an ISL ladder; the expanded view shows the cut-out designed for easy access for microbonding. On the right you see the ISL Mounting Jig.

alignment of a ladder has to be much better than the intrinsic hit resolution of $25 \mu \mathrm{m}$ (see Section 5.1). We achieve this high precision with one rotating stage, holding the hybrid, and a row of $3 X-$, $Y$-rotating stages, holding the sensors (Fig. 3 right). The hybrid and the three sensors are held in position by applying a vacuum. The teflon surfaces of the vacuum chucks on top of the towers have an accuracy in planarity of $\pm 5 \mu \mathrm{m}$. The carbon fiber support is placed on a metal bridge underneath the teflon vacuum chucks, led by high precision bearings to provide good planarity. The mounting is done on a coordinate measurement machine $(\mathrm{CMM})$ with a precision along the strips of about $\pm 1 \mu \mathrm{m}$. The hybrid is mounted first, rotated with respect to the carbon fiber ladder, the three silicon sensors are then positioned with respect only to the precision hole on the hybrid. The glue-laden ladder is then elevated to the sensors. The structure is released when the glue has fully settled. This procedure ensures an internal module precision only depending on the CMM precision for construction. It ensures an internal precision independent of the carbon fiber ladder. The wire bonding to connect the sensors is done after mounting, therefore the bonding areas have to be fully accessible after gluing (Fig. 3, left). Finally the two ladders are glued together on a similar mounting fixture to form a module. The precision is controlled by a CMM.

\subsection{Spaceframe}

The spaceframe was designed with respect to weight, stiffness and material budget, which led to a carbon fiber structure. Carbon fiber rings are connected with hollow carbon fiber rods. The total weight of the structure is about $6 \mathrm{~kg}$ before mounting the modules. Finite element analysis predicts a maximum gravitational sag of less than $30 \mu \mathrm{m}$ in the center. We expect the structure to be even stiffer after mounting the modules, with a final precision of $50 \mu \mathrm{m}$ over the whole length of $2 \mathrm{~m}$. 


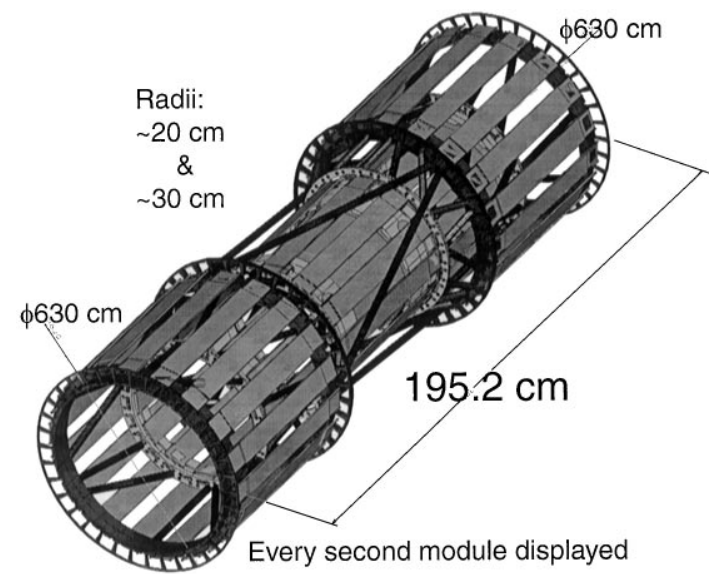

Fig. 4. Mechanical design of the carbon spaceframe. The geometrical concept of the single additional layer in the central region and the two additional layers in the forward direction can be seen.

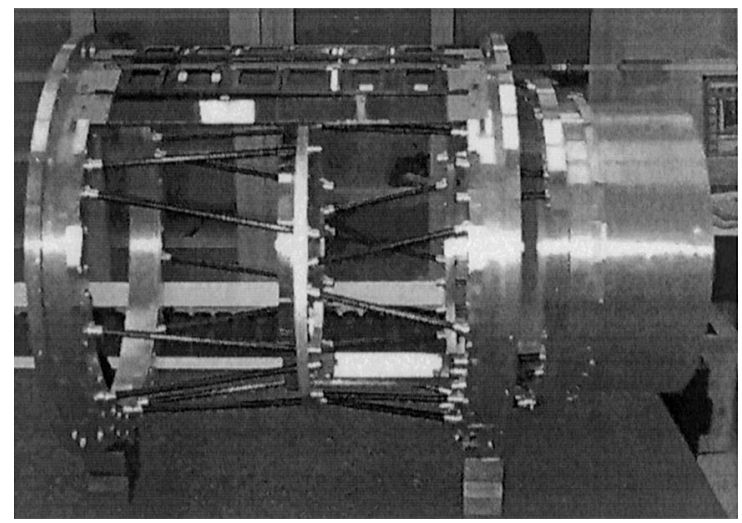

Fig. 5. Picture of the aluminium prototype. One forward prototype barrel is displayed with the final hollow carbon fiber rods. Some dummy modules are mounted to check the assembly procedure.

The internal module precision is ported to the final position precision in the spaceframe by only connecting the hybrids, not the carbon fiber ladders. The position of each silicon sensor is measured by a CMM. The final alignment precision will be achieved with tracks (see Figs. 4 and 5).

\subsection{Assembly}

The spaceframe was designed in Pisa, and carbon fiber parts are laminated by Monfrini S.R.L. (Italy).



Fig. 6. Assembly of the SVX II and the ISL on one CMM. We use three granite tables, with a CMM on the inner one to support a rail system supporting both ISL and SVX II. Everything will be assembled on a rail system, which itself will be used to insert both detectors into the COT.

The gluing of single parts into the whole structure will be done in Pisa under a CMM using precision gluing masks to obtain the needed accuracy. Struts are custom-made by Reglass S.p.A. (Italy). The spaceframe is designed in such a way that it can be disassembled into three parts and shipped to Fermilab. The SVX II and the ISL will be assembled together on the adapted rail system, previously used to install the SVX' in RUN I. We make use of one single CMM by placing one granite table on both ends. SVX II and ISL will then be placed on the rail system, resting on this set of granite tables (see Fig. 6). We will begin to assemble the SVX II on the CMM, then move it to one side to make room for the ISL. After assembling both detectors, we will mount the SVX II in the ISL. The same rail system will also be used to insert the ISL and SVX II into the COT. All parts rest on the same rail from the beginning of assembly until final insertion into CDF II.

\section{Electrical components}

\subsection{Silicon detectors $[5-7]$}

The sensors are $7.4 \times 5.5 \mathrm{~cm}^{2}$ silicon microstrip detectors, $300 \mu \mathrm{m}$ thick, AC coupled with 512 axial strips on the p-implant side and 512 stereo strips $\left(1.2^{\circ}\right)$ on the n-implant side; both sides have a strip pitch of $112 \mu \mathrm{m}$. The sensors consist of high resistivity n-bulk silicon. We use a conventional design: bias is applied via polysilicon resistors, one guardring for field shaping and for the n-side a p-stop design is used. Detectors will be produced by Micron Semiconductors on 6" wafers. A slightly 
shorter version $\left(6.7 \times 5.7 \mathrm{~cm}^{2}\right)$ of the same detectors will be manufactured by Hamamatsu Photonics on $4^{\prime \prime}$ technology, with the stereo strips $\left(1.2^{\circ}\right)$ on the $p$ implant side. The performance of Micron prototypes was measured in a testbeam. Using the SVX electronics, we measured a resolution of $25 \mu \mathrm{m}$ with an average signal/noise ratio of 21 for an average cluster size of 1.7 strips for one detector (with no magnetic field) [4]. The prototype phase is finished and Micron has started production. Each sensor is sent to Karlsruhe for quality control, before the final ladder assembly in Pisa. The quality control checks for pinholes and strips with a high leakage current. We require less than $3 \%$ bad channels in total and less than $2 \%$ on each side.

\subsection{The SVX3 chip $[1,3]$}

The silicon sensors are read out by the SVX3 chip, a radiation hard CMOS custom integrated circuit. Each chip has 128 parallel input channels. The chip consists of two separate parts: the frontend for the analog functions and the backend part for the digital functions. The input amplifiers, integrators, the 46 cell pipelines needed for deadtimeless operation and the pipeline acquisition logics are located in the frontend. Up to 4 groups of cells can be queued for digitization and readout at one time. The backend consists of an 8 bit Wilkinson ADC, a readout FIFO and differential output drivers. The chip is able to work with positive and negative signals to handle double sided silicon detectors. It has a sparsification mode, a calibration input and is capable of pedestal subtraction on chip level. The chip is programmable to adjust for different Tevatron running conditions. e.g bunch-spacings (132-396 ns). It can handle strip capacitances from 10 to $35 \mathrm{pF}$. Also the pipeline depth, threshold level and bandwidth limit is adjustable. The chip has been irradiated with a $\mathrm{Co}^{60}$ source up to $4 \mathrm{Mrad}$ and was found to perform well ${ }^{2}$ [8]. The amplification is $15 \mathrm{mV} / \mathrm{fC}$, which corresponds to $60 \mathrm{mV}$ for a minimum ionizing particle. The chip provides

\footnotetext{
${ }^{2}$ During RUN II the chip will be exposed to about $7 \times 10^{13}$ minimum ionizing particles per $\mathrm{cm}^{2}$ over a period of two years, corresponding to an absorbed dose of $2 \mathrm{Mrad}$ at the inner layer of SVX II [9].
}

deadtimeless readout with Level-1 trigger rates up to $50 \mathrm{kHz}$. The maximum delay between Level-1 trigger decision and readout without overwriting the pipeline cell content is $5.5 \mu$ s at 132 ns between beam crossings.

\section{The on-line alignment system $[10,11]$ and active alignment}

The Silicon Vertex Trigger (SVT) will use impact-parameter information in the trigger to detect secondary vertices. In order to obtain impact-parameter information in the Level-2 trigger, the SVX II is read out after each Level-1 trigger. The SVT combines this data with the Level-1 tracking information from the COT, reconstructed with the XFT (eXtremely Fast Tracker) trigger [1]. The SVT computes track parameters in the transverse plane $\left(\phi_{0}, p_{\mathrm{T}}\right.$, and impact parameter) with resolution and efficiency comparable to full offline analysis. This trigger needs the beam axis to be parallel to the tracking system. Small tilts can be corrected for by adjusting the Tevatron beam. For this it is necessary to feed back the beam position to the Tevatron control room. To adjust for larger tilts an active alignment system is foreseen. It allows to move and tilt the SVX II/ISL with respect to the beam. The movement is achieved by mounting the system on rods. Micro motors (so called "inchworms" based on piezo crystals) move along the rods with high precision and so vary the position of the SVX II/ISL system. The relative positions of SVX II, ISL and COT will be continuously monitored by the so called Rasnik system ${ }^{3}$ and controlled. A system of a light source, consisting of an array of 9 infrared LEDs, a distinctive pattern, a lens and a CCD camera will be mounted in the detector. The pattern is continuously projected on the CCD. Any relative movement of LED-array and CCD will be noticed as a shift of the pattern. A PC with pattern recognition software will calculate the movement and rotation with a precision in the submicron range. It is also suited to detect

\footnotetext{
${ }^{3}$ A similar system was used in the CHORUS experiment.
} 




Fig. 7. The online global alignment system. This picture displays the spaceframes of SVX II, ISL, COT and eight Rasnik systems. With this arrangement the relative positions of the 3 subdetectors can be monitored. One control unit consists of an array of infrared LEDs as source, a projected pattern, a lens to focus the light and the CCD as receptor. Each change of position of source or camera will be observed as a shift of the pattern.

changes due to temperature and humidity changes in the whole structure. In Fig. 7 is shown the Rasnik components positions in the system; relative shift of ISL to SVX II, ISL to COT, twists in the SVX II and the global tracking system to endflange position can be monitored.

\section{Conclusion}

The ISL project started in late 1996, as part of the CDF upgrade for RUN II. The ISL-SVX II combination forms a standalone silicon tracking system and extends the tracking region from $|\eta|<1$ up to $|\eta| \leq 2$. Adding the ISL in the central area $|\eta|<1$ improves the tracking precision, it also helps to resolve ambiguities in dense track environments. The spaceframe design is finished and a prototype has been built. The mounting fixtures have been manufactured and exceed the expected precision. The silicon sensors passed the design and prototype phase and production started. The hybrids are designed and the first hybrids were produced. The first prototype ladder has been built and is working. We expect the ISL-SVX II tracking system to be ready for installation in November 1999.

\section{References}

[1] CDF II Collaboration, Technical Design Report, FERMILAB-Pub-96/390-E, 1996.

[2] D0 Collaboration, Nucl. Instr. and Meth. A 338 (1994) 185, FERMILAB-PUB-93/179-E.

[3] Maurice Garcia-Sciveres, Nucl. Instr. and Meth. A 435 (1999) 58.

[4] Detector prototype test for ISL, CDF INTERNAL NOTE 4450.

[5] Frank Hartmann et al., Evaluation of the ISL prototypes, CDF Internal Note 4627.

[6] Gino Bolla, Nucl. Instr. and Meth. A 435 (1999) 51.

[7] K. Hara et al., 4" prototype Si microstrip sensors for the CDF ISL, CDF Internal Note 4506.

[8] Maurice Garcia-Sciveres et al., Radiation effects on the SVX3 chip, CDF Internal Note 4461.

[9] Barnett ., (Particle Data Group), Phys. Rev. D 54 (1996) 1268.

[10] D. Saltzberg et al., The optical alignment monitoring system of CHORUS (RASNIK), CERN-PPE/96-179.

[11] Eddie Sanders, Rasnik relative alignment monitor for the CDF II SVX II-ISL-COT, CDF Internal Note 4510. 